\title{
Methodische Ansätze zur Cr(III)/Cr(VI)-Speciesanalyse mit Hilfe der elektrothermalen Atomabsorptionsspektrometrie
}

\author{
K. Dungs, H. Fleischhauer und B. Neidhart
}

Institut für Arbeitsphysiologie an der Universität Dortmund, Ardeystr. 67, D-4600 Dortmund, Bundesrepublik Deutschland

Methodical developments for the speciation of $\mathrm{Cr}(\mathrm{III}) / \mathrm{Cr}(\mathrm{VI})$ by electrothermal atomic absorption spectrometry

Fresenius Z Anal Chem (1985) 322:280-289

Seite 281, Tabelle 1 lautet richtig:

Tabelle 1. Beispiele für häufig verwendete Reagentien in der GOCTechnik

\begin{tabular}{|c|c|c|c|c|}
\hline \multirow[t]{2}{*}{ Element } & \multirow[t]{2}{*}{ Modifier } & \multicolumn{2}{|c|}{$\begin{array}{l}\text { Maximale Veraschungs- } \\
\text { temperatur }\left({ }^{\circ} \mathrm{C}\right)\end{array}$} & \multirow[t]{2}{*}{ Literatur } \\
\hline & & $\begin{array}{l}\text { ohne } \\
\text { Modifier }\end{array}$ & $\begin{array}{l}\text { mit } \\
\text { Modifier }\end{array}$ & \\
\hline $\mathrm{Al}$ & A & 1500 & 1700 & {$[3,4,5,24]$} \\
\hline As & $\mathrm{C}$ & 300 & $1300^{\circ}$ & {$[2,6,7,17]$} \\
\hline $\mathrm{Bi}$ & $\mathrm{C}$ & 500 & 1200 & [18] \\
\hline $\mathrm{Cd}$ & $\mathrm{B}, \mathrm{F}, \mathrm{G}, \mathrm{H}, \mathrm{I}$ & 250 & 900 & $\begin{array}{l}{[2,8,9,16,} \\
21,22]\end{array}$ \\
\hline $\mathrm{Co}$ & $\mathrm{A}$ & 1000 & 1400 & {$[9,10,11,24]$} \\
\hline $\mathrm{Cr}$ & $\mathrm{A}$ & 1200 & 1650 & {$[9,12,24]$} \\
\hline $\mathrm{Cu}$ & I & 1200 & 1200 & {$[16]$} \\
\hline $\mathrm{Hg}$ & $\mathrm{J}, \mathrm{K}, \mathrm{L}$ & - & $150-300$ & $\begin{array}{l}{[2,13,14,25,} \\
26]\end{array}$ \\
\hline $\mathrm{Mn}$ & $\mathrm{A}$ & 1000 & 1400 & {$[9,14]$} \\
\hline $\mathrm{Ni}$ & $A, B$ & 1000 & 1400 & {$[9,21,23]$} \\
\hline $\mathrm{Pb}$ & $\mathrm{B}, \mathrm{M}$ & 500 & 950 & {$[9,21,23]$} \\
\hline $\mathrm{Se}$ & $\mathrm{D}, \mathrm{K}, \mathrm{S}$ & 200 & $1100-1300$ & {$[9,20,26]$} \\
\hline $\mathrm{Te}$ & $\mathrm{D}, \mathrm{O}, \mathrm{P}, \mathrm{Q}$ & 600 & 1050 & {$[14,19]$} \\
\hline $\mathrm{Zn}$ & $\mathrm{B}, \mathrm{R}$ & 400 & $900^{\circ}$ & {$[14,27]$} \\
\hline
\end{tabular}

$\mathrm{A}, \mathrm{Mg}\left(\mathrm{NO}_{3}\right)_{2} ; \mathrm{B}, \mathrm{NH}_{4} \mathrm{H}_{2} \mathrm{PO}_{4} ; \mathrm{C}, \mathrm{Ni} ; \mathrm{D}, \mathrm{Cu} ; \mathrm{F},\left(\mathrm{NH}_{4}\right)_{2} \mathrm{SO}_{4} ; \mathrm{G}$, $\left(\mathrm{NH}_{4}\right)_{2} \mathrm{~S}_{2} \mathrm{O}_{8} ; \mathrm{H}, \mathrm{NH}_{4} \mathrm{~F} ; \mathrm{I}, \mathrm{O}_{2} ; \mathrm{J},\left(\mathrm{NH}_{4}\right)_{2} \mathrm{~S}$; $\mathrm{K}, \mathrm{K}_{2} \mathrm{Cr}_{2} \mathrm{O}_{7} ; \mathrm{L}, \mathrm{H}_{2} \mathrm{O}_{2}$; M, La; O, Pd; P, Pt; Q, $\left(\mathrm{NH}_{4}\right)_{2} \mathrm{Cr}_{2} \mathrm{O}_{7} ; \mathrm{R}, \mathrm{H}_{3} \mathrm{PO}_{4} ; \mathrm{S}, \mathrm{Mo}$
Seite 282, linke Spalte „Chemikalien“:

die drei letzten Positionen müssen lauten:

- Natriumacetat, p.a. ${ }^{1}$;

- Harnstoff, p.a. ${ }^{1}$

- Lanonorm Metalle $2^{4}$.

Seite 285, Tabelle 5 lautet richtig:

Tabelle 5. Optimiertes Temperaturprogramm für die $\mathrm{Cr}(\mathrm{III}) / \mathrm{Cr}(\mathrm{VI})$ Speciestrennung mit der ETAAS-Technik

\begin{tabular}{|c|c|c|c|c|c|c|c|c|}
\hline & \multicolumn{8}{|c|}{ Step } \\
\hline & 1 & 2 & 3 & 4 & 5 & 6 & 7 & 8 \\
\hline Temp. $\left({ }^{\circ} \mathrm{C}\right)$ & 80 & 150 & 220 & 500 & 1000 & 2400 & 2650 & 20 \\
\hline Ramp (s) & 5 & 15 & 25 & 10 & 1 & 0 & 1 & 1 \\
\hline Hold (s) & 50 & 70 & 40 & 10 & 10 & 6 & 4 & 20 \\
\hline Read & & & & & & -1 & & \\
\hline Rec. & & & & & & -1 & & \\
\hline Int. Flow & & & & & & 50 & & \\
\hline
\end{tabular}

Seite 288, Literatur: die Stelle 15 ist ersatzlos zu streichen. 